\title{
Qualitative dynamics of planar chains
}

\author{
S. Laederich ${ }^{\mathrm{a}, 1}$ and M. Levi ${ }^{\mathrm{b}, 2}$ \\ ${ }^{a}$ University of Michigan, Ann Arbor, MI 48109, USA \\ ${ }^{\mathrm{b}}$ Department of Mathematical Sciences, Rensselaer Polytechnic Institute, Troy, NY 12180-3590, USA
}

\author{
Received 9 February 1990 \\ Revised manuscript received 23 October 1990 \\ Accepted 29 October 1990 \\ Communicated by $\mathrm{H}$. Flaschka
}

\begin{abstract}
In this paper we derive the equations of motion and analyze some aspects of the dynamics of planar chains. We show that (a) for any integer vector $n=\left(n_{1}, \ldots, n_{N}\right)$ (where $N$ is the number of joints) there exists a periodic motion such that the angle of $j$ th joint changes by $2 \pi n_{j}$ during one period; (b) the straight configuration is (nonlinearly) stable and that any "folded" rectilinear configuration is unstable; (c) the Coriolis effects play no role in stability of these rectilinear configurations, in contrast to other problems such as the stabilizing effect on the spinning top.
\end{abstract}

\section{Introduction and results}

In this paper we derive the equations of motion and study the dynamics of planar chains consisting of coupled rigid bodies, in the absence of external forces. More precisely, we consider $N+2$ equal point masses $m=1 /(N+2)$ of total mass one, coupled by $N+1$ massless links of length $l$.

Our subsequent discussion carries over almost verbatim to the case of unequal masses and lengths. We can allow torsional coupling between the links, in particular the nearest neighbor coupling. In the latter case we obtain precisely the discretization of the beam, and thus the exact equations for such a beam. It is a crucial point that we derive the equations in intrinsic coordinates of the chain, i.e. without any reference to

\footnotetext{
${ }^{1}$ Supported in part by the Forschungs Institut für Mathematik, ETH Zürich. Current address: IMA, University of Minnesota.

${ }^{2}$ Supported in part by AFOSR grant 0.144-85. Permanent address: Department of Mathematics, Boston University, 111 Cummington Street, Boston, MA 02215, USA.
}

an inertial frame. It is very useful to have such reduced equations for numerical simulations, since the numerical damping does not affect the angular momentum of the chain as it does when the non-reduced equations are used.

We use some standard methods in the calculus of variations in the large to describe an interesting class of periodic motions of the system. The variational methods have been used in showing the existence of periodic solutions in many settings; of the more recent examples we mention, without an attempt at completeness, refs. [8, 9, $11,13]$. We will also prove nonlinear stability of some simple motions for all time.

The problem of dynamics of coupled rigid bodies has been studied at least since the last century, but in recent years it has received renewed attention due to its relevance in understanding the dynamics of flexible space structures as well as in some applications in robotics; see refs. [2, 4, $5,10,14,17,18]$.

The system of compound pendula that was studied extensively $[1,12,13]$ is somewhat similar 


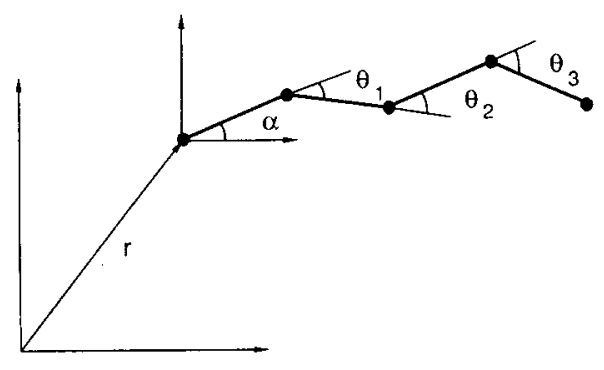

Fig. 1. The Chain.

to ours in that it is just a chain suspended by one end in a gravitational field. The crucial difference between this system and the free chain considered here is the lack of a preferred inertial frame for the free chain. It is therefore desirable to reduce the dimension of the problem by eliminating the reference to an inertial frame; the reduced equations will be in terms of angles $\boldsymbol{\theta}_{j}$ between the neighboring links (fig. 1) and will not contain any variables characterizing the orientation or position with respect to an inertial frame.

We will show that the evolution of the angles $\theta_{j}$ is governed by the Euler-Lagrange equations with a Lagrangian of the form $L=L_{2}+L_{1}+L_{0}$, where $L_{i}$ is homogeneous of degree $i$ in terms of the relative angular velocities $\dot{\theta}_{j}, j=1, \ldots, N$. The term $L_{1}$ gives rise to the gyroscopic (or Coriolis) forces. In the case when $L_{1}=0$, i.e. when $L=$ $L_{2}+L_{0}$, the Maupertuis least action principle states that the trajectories with energy $E$ connecting two points $\theta^{0}$ and $\theta^{1}$ in the configuration space are the extremals of

$\int_{0}^{1} \sqrt{\left(L_{0}+E\right) L_{2}} \mathrm{~d} t$

and thus the trajectories are the geodesics for the Riemannian metric given by $\mathrm{d} J^{2}=\left(L_{0}+\right.$ E) $L_{2} \mathrm{~d} t^{2}$; it is known as the Jacobi metric. In the presence of gyroscopic forces, i.e. when $L_{1} \neq 0$, the Maupertuis principle is modified to

$\delta \int_{0}^{1}\left[2 \sqrt{\left(L_{0}+E\right) L_{2}}+L_{1}\right] \mathrm{d} t=0$ see refs. [5, 13] and $\mathrm{d} J^{2}=\left[2 \sqrt{\left(L_{0}+E\right) L}+\right.$ $\left.L_{1}\right] \mathrm{d} t^{2}$ is no longer a metric since $L_{1}$ changes sign when $\dot{\theta}$ is replaced by $-\dot{\theta}$. This can be restated by saying that the indicatrix

$$
\left\{\dot{\theta}: 2 \sqrt{\left(L_{0}+E\right) L_{2}}+L_{1}=\text { const., } \theta \text { fixed }\right\}
$$

is non-symmetric with respect to the origin $\dot{\theta}=0$ in the tangent space, and the dynamical system is no longer reversible.

In order to obtain periodic solutions one wishes to apply, for instance, a curve shortening process to a noncontractible curve on the configuration torus $\left\{\theta=\left(\theta_{1} \bmod 2 \pi, \ldots, \theta_{N} \bmod 2 \pi\right)\right\}$. Precisely this method has been used since Hadamard [6, 20], but only for the case of a Riemannian metric. In this paper we point out that the same ideas and methods go through in the case of a nonreverisble "metric" as well. As a result we obtain the following

Theorem 1.1. For any value of the angular momentum $\mu$ there exists an energy value $E_{0}(\mu)$ such that for any energy value $E>E_{0}(\mu)$ there are periodic motions of the following kind. Given any $N$-tuple of integers $\boldsymbol{n}=\left(n_{1}, \ldots, n_{N}\right)$, there exists a periodic motion $\theta(t)$ of the chain (with energy and momentum $E$ and $\mu$ as prescribed above) of period $T=T(E, \mu, n)$ such that during time $T$ the $i$ th angle $\theta_{i}$ makes exactly $n_{i}$ full turns; more precisely, there exists a solution $\theta(t)$ with $\theta(T)-\theta(0)=2 \pi n, \dot{\theta}(T)=\dot{\theta}(0)$. In other words, the numbers $n_{i}$ of relative revolutions between two neighbors can be prescribed arbitrarily and for some initial conditions they will in fact be realized. Viewed from an inertial frame these motions are either periodic or quasiperiodic.

We also prove the following stability result.

Theorem 1.2. For any non-zero value $\mu_{0} \neq 0$ of the total angular momentum, the straight line configuration $\theta(t) \equiv 0$ of the chain is stable; any 
folded configuration (at least one $\theta_{i}=\pi$ and $\theta_{i}=$ 0 or $\pi$ ) is unstable. More precisely, given any $\epsilon>0$ there exists $\delta=\delta(\mu)>0$ such that for all initial conditions with $|\theta|+|\dot{\theta}|<\delta$ for $t=0$ we have $|\theta|+|\dot{\theta}|<\epsilon$ for all $t \in \mathbb{R}$. Moreover, $\epsilon$ and $\delta$ need not be small; they are given by explicit estimates (4.4) and (4.5) below.

The stability of the straight configuration is intuitively rather clear. The proof, however, turns out to be a little less trivial than one might expect, and we provide it for completeness. As a byproduct of the proof, we get quantitative estimates on the stability region (i.e. on $\epsilon$ and $\delta$ ).

\section{Derivation of the equations of motion}

\subsection{The model}

We consider $N+2$ masses $m$ linked together by $N+1$ massless links of length $l$, restricted to a plane. In this section we derive the equations of motion in intrinsic coordinates without reference to an inertial frame. We choose the angles $\theta_{j}$ between the $j$ th and $(j+1)$ th segment as such coordinates, see fig. 1.

The equations of motion turn out to be simpler when written in terms of the cumulative angles $\phi_{j}=\sum_{k-1}^{j} \theta_{k}$; the $N$-tuple of these angles will be denoted by $\phi=\left(\phi_{1}, \phi_{2}, \ldots, \phi_{N}\right)$.

\subsection{Equations of motion}

In the absence of external forcing the angular momentum $\mu$ is conserved. We have

Theorem 2.1. The equation of motion of the system are given by the Lagrangian

$$
\begin{aligned}
L(\phi, \dot{\phi})= & \frac{1}{2}(K(\phi) \dot{\phi}, \dot{\phi})+\mu \omega(\phi) \cdot \dot{\phi} \\
& -\frac{1}{2} \mu^{2} / I(\phi),
\end{aligned}
$$

where $K(\phi)$ is a positive definite matrix, periodic in $\phi, \omega(\phi)$ is a $N$-vector and $I(\phi)$ is the moment of inertia relative to the center of mass of the system. All these objects are given via a single matrix $Q$ in (2.2), (2.3) below.

The so-called gyroscopic term $\omega$ in the Lagrangian gives rise to the Coriolis force; the notation $\omega$ is suggested by the analogous expression $2 \omega \times \dot{x}$ for the Coriolis force in the case of a single particle at $x$ in a frame rotating with the angular velocity $\omega$.

\subsection{Expressions for $K(\phi), \omega(\phi)$ and $I(\phi)$}

The quantities $K, \omega$ and $I$ admit an elegant expression in terms of a single $(N+1) \times(N+1)$ matrix $Q$. For any $\psi \in \mathbb{R}^{N+1}$ define, for $j, k=$ $0, \ldots, N$,

$$
\begin{aligned}
Q_{j k}(\psi)= & l^{2}\left(\frac{N+1-\max (j, k)}{N+2}\right. \\
& \left.-\frac{N+2-(N+1-j)(N+1-k)}{(N+2)^{2}}\right) \\
& \times \cos \left(\psi_{j}-\psi_{k}\right) .
\end{aligned}
$$

Denoting by $\Phi$ the vector $(0, \phi) \in \mathbb{R}^{N+1}$, we will derive the following expressions for $K(\phi), \omega(\phi)$ and $I(\phi)$ :

$$
(K(\phi) \dot{\phi}, \dot{\phi})=(Q(\Phi) \dot{\Phi}, \dot{\Phi})-\frac{(Q(\Phi) \dot{\Phi}, 1)^{2}}{(Q(\Phi) I, I)}
$$

$\omega(\phi) \cdot \dot{\phi}=\frac{(Q(\Phi) \dot{\Phi}, I)}{(Q(\Phi) I, I)}$

and

$I(\phi)=(Q(\Phi) 1,1)$,

where $I=(1, \ldots, 1)(N+1$ times $)$.

\subsection{Derivation of the reduced Lagrangian}

We start with the full Lagrangian which is just the kinetic energy expressed in an inertial frame. 
Using the complex numbers to denote the position of a point in the plane, we let $r$ be the position of the first mass point relative to a chosen inertial frame. Let $\alpha$ be the angle of the first link with the $x$-axis, and let

$z_{n}=l \mathrm{e}^{\mathrm{i} \alpha} \sum_{j=1}^{n} \mathrm{e}^{\mathrm{i} \phi_{j-1}}, \quad$ where $\Phi=(0, \phi)$,

be the position of the $n$th mass point relative to the first mass point. The Lagrangian is then expressed as the sum of the kinetic energies of all masses:

$\mathscr{L}(\dot{r}, \alpha, \dot{\alpha}, \phi, \dot{\phi})=\frac{1}{2} m \sum_{n=0}^{N+1}\left|\dot{r}+\dot{z}_{n}\right|^{2}$.

We will obtain the reduced Lagrangian (2.1) by the Routh reduction, eliminating $r$ and $\alpha$. By Noether's theorem [1], the conservation of linear momentum and the conservation of angular momentum can be written as

$\mathscr{L}_{\dot{r}}=\lambda$,

$r \wedge \mathscr{L}_{\dot{r}}+\mathscr{L}_{\dot{\alpha}}=\mu$,

where $\wedge$ denotes the scalar cross product: $z \wedge$ $w=\operatorname{Im}(\bar{z} \mathrm{w})$. Without loss of generality, we assume that the linear momentum $\lambda$ vanishes.

Introducing the positions

$p_{n}=\mathrm{e}^{-\mathrm{i} \alpha} z_{n}$

and their average

$\langle p\rangle=\frac{1}{N+2} \sum_{n=1}^{N+1} p_{n}$,

we obtain from the conservation laws (2.5) with $\lambda=0$

$$
\begin{aligned}
& \dot{r}=-\mathrm{i} \alpha \mathrm{e}^{i \alpha}\langle p\rangle-\mathrm{e}^{\mathrm{i} \alpha}\langle\dot{p}\rangle, \\
& \dot{\alpha}=\frac{\mu-(\langle p \wedge \dot{p}\rangle-\langle p\rangle \wedge\langle\dot{p}\rangle)}{\left\langle|p|^{2}\right\rangle-|\langle p\rangle|^{2}} .
\end{aligned}
$$

The denominator of $(2.8 \mathrm{~b})$ is precisely the moment of inertia $I(\phi)$. It never vanishes since it is equal to

$$
\frac{1}{N+2} \sum_{n=1}^{N+1}\left|p_{n}-\langle p\rangle\right|^{2}
$$

We can now use the Routh reduction $[19,21]$ to obtain the reduced Lagrangian $L$. Denote by $\overline{\mathscr{L}}(\phi, \dot{\phi}, \mu)$ the Lagrangian $\mathscr{L}$ given by $(2.4)$ in which $\dot{r}$ and $\dot{\alpha}$ have been replaced according to (2.8); it is important that $\mathscr{L}$ is a $\alpha$-independent. Using the fact that along a solution $\mathscr{L}_{\dot{r}}=0$ and $\mathscr{L}_{\dot{\alpha}}=\mu$, one has the identities in $t$

$\overline{\mathscr{L}}_{\phi}=\mathscr{L}_{\phi}+\mu \dot{\alpha}_{\phi}, \quad \tilde{\mathscr{L}}_{\phi}=\mathscr{L}_{\phi}+\mu \dot{\alpha}_{\phi}$

Hence solutions with $\lambda=0$ of the EulerLagrange equations

$\frac{\mathrm{d}}{\mathrm{d} t} \mathscr{L}_{\dot{\phi}}-\mathscr{L}_{\phi}=0$

satisfy

$\frac{\mathrm{d}}{\mathrm{d} t}\left(\tilde{\mathscr{L}}_{\dot{\phi}}-\mu \dot{\alpha}_{\dot{\phi}}\right)-\left(\tilde{\mathscr{L}}_{\phi}-\mu \alpha_{\phi}\right)=0$,

i.e. they satisfy the Euler-Lagrange equations

$\frac{\mathrm{d}}{\mathrm{d} t} L_{\dot{\phi}}-L_{\phi}=0$

for the new Lagrangian

$L(\phi, \dot{\phi}, \mu)=\tilde{\mathscr{L}}(\phi, \dot{\phi}, \mu)-\mu \dot{\alpha}(\phi, \dot{\phi}, \mu)$

With a little algebra one obtains

$$
\begin{aligned}
& L(\phi, \dot{\phi}, \mu)=\frac{1}{2}\left(\left\langle|\dot{p}|^{2}\right\rangle-|\langle\dot{p}\rangle|^{2}\right) \\
& -\frac{1}{2} \frac{(\mu-(\langle p \wedge \dot{p}\rangle-\langle p\rangle \wedge\langle\dot{p}\rangle))^{2}}{\left\langle|p|^{2}\right\rangle-|\langle p\rangle|^{2}} .
\end{aligned}
$$


Using the identities

$$
\begin{aligned}
& \sum_{n=1}^{N} \sum_{k=1}^{n} a_{k}=\sum_{k=1}^{N}(N+1-k) a_{k}, \\
& \sum_{n=1}^{N} \sum_{j=1}^{n} \sum_{k=1}^{n} a_{j k} \\
& \quad=\sum_{j=1}^{N} \sum_{k=1}^{N}[N+1-\max (j, k)] a_{j k}, \\
& a_{j k}=a_{k j},
\end{aligned}
$$

one immediately obtains that for $\Phi=(0, \phi)$, in the above notation (2.2) for $Q$ (expressed in terms of $\Phi$ )

$$
\begin{aligned}
& \left\langle|\dot{p}|^{2}\right\rangle-|\langle\dot{p}\rangle|^{2}=(Q(\Phi) \dot{\Phi}, \dot{\Phi}), \\
& \langle|p|\rangle-|\langle p\rangle|^{2}=I(\Phi)=(Q(\Phi) I, I), \\
& \langle p \wedge \dot{p}\rangle-\langle p\rangle \wedge\langle\dot{p}\rangle=(Q(\Phi) \dot{\Phi}, l) .
\end{aligned}
$$

It remains to show that

$$
(Q(\Phi) \dot{\Phi}, \dot{\Phi})-\frac{(Q(\Phi) \dot{\Phi}, 1)^{2}}{(Q(\Phi) 1,1)}=\mathscr{K}(\Phi, \dot{\Phi})
$$

is a positive definite quadratic form in $\dot{\phi}$. Consider the following polynomial in $\lambda$ :

$$
\begin{aligned}
& \lambda^{2}(Q(\Phi) \dot{\Phi}, \dot{\Phi})-\lambda(Q(\Phi) \dot{\Phi}, 1)+(Q(\Phi) 1,1) \\
& \quad=(Q(\Phi)(\lambda \dot{\Phi}+1),(\lambda \dot{\Phi}+1)) .
\end{aligned}
$$

Its discriminant is equal to $-\frac{1}{4}(Q(\Phi) 1,1)$ $\times \mathscr{K}(\Phi, \dot{\Phi})$. Since $Q$ is positive definite the polynomial can only have roots for $\dot{\Phi}=-(1 / \lambda) 1$. Since the first component of $\dot{\Phi}$ is always equal to zero, the last equality cannot hold so that the polynomial has no real roots and hence the discriminant is negative, thus implying the positivity of $\mathscr{K}(\Phi, \dot{\Phi})$.

QED

\section{Proof of the existence of periodic solutions}

For an arbitrarily chosen integer vector $n=$ $\left(n_{1}, \ldots, n_{N}\right)$ we choose a smooth oriented loop $\theta(s), 0 \leq s \leq 1$ on the torus $\mathrm{T}^{N}=\{\theta \bmod 2 \pi\}$ with $\theta(1)-\theta(0)=2 \pi n$, and subject this loop to the curve shortening process. This construction is usually done for a Riemannian metric as in ref. [5] or ref. [20] but the arguments carry over verbatim to the case of the Maupertuis pseudometric

$\mathrm{d} J^{2}=\left[2 \sqrt{\left(L_{0}+E\right) L_{2}}+L_{1}\right]^{2} \mathrm{~d} t^{2}$,

where $L=L_{0}+L_{1}+L_{2}$ is the Lagrangian given by (2.1) and where the $L_{j}$ are homogenous of degree $j$ in the velocities. The only difference with the metric case is the non-reversibility. The shortest path (and thus the distance) from $A$ to $B$ differs from that from $B$ to $A$. In the rest of the discussion, "distance" refers to the directed distance in the direction of the orientation of the curves.

There exists $E_{0}=E_{0}(\mu)$ such that for all $E>$ $E_{0}(\mu)$ the Maupertuis pseudo-metric $\mathrm{d} J^{2}$ is positive (i.e. the gyroscopic term $L_{1}$ is dominated by the positive term $\sqrt{\left.\left(L_{0}+E\right) L_{2}\right)}$. For any such $E>E_{0}(\mu)$ which we fix from now on, there exists an injectivity radius $\rho>0$, i.e. the number for which any two points $A$ and $B$ at a distance (measured in $J$ ) less than $\rho$ can be connected by a unique minimal from $A$ to $B$, that is, an extremum of

$J(\gamma)=\int_{\gamma} \mathrm{d} J, \quad \gamma(0)=\mathrm{A}, \quad \gamma(1)=\mathrm{B}$

We can now carry out the curve shortening procedure. We equipartition the curve $\theta$ into $q$ equal segments. We denote all the partition points (on the covering space of $\mathrm{T}^{N}$ ) by $\theta_{j}$, where $\theta_{j+q}=$ $\theta_{j}+2 \pi n$ and $\operatorname{dist}\left(\theta_{j}, \theta_{j+1}\right)=q^{-1}$ length $(\theta)$. All distances will be counted in the direction of increasing $s$. We choose $q$ so that the length of $\theta$ is less or equal to $q \rho$. One then replaces the curve segments between $\theta_{j}$ and $\theta_{j+1}$ by minimals of the variational principle. Note that this will reduce 
the length of the curve exept when each segment between $\theta_{j}$ and $\theta_{j+1}$ is minimal. We then take the midpoints $\mathbf{M}_{j}$ of the new segments and connect $\mathbf{M}_{j}$ and $\mathbf{M}_{j+1}$ by a minimal. This second step also reduces the total length except if the first step yielded an extremal of the variational principle. As in ref. [20], one can show that the curve obtained from $\theta$ by this procedure is a continuous deformation of $\theta$, hence of the same homotopy type.

Repeating this procedure yields a sequence of curves $\theta^{(k)}$ of decreasing length and of the same homotopy type. Note that by construction, each of these curves is determined by $q$ points $\theta_{j}^{(k)}$ since neighbouring points $\theta_{j}^{(k)}$ and $\theta_{j+1}^{(k)}$ are connected by a unique minimal. Since the set of $q$-tuples of points of $\mathrm{T}^{n}$ is compact, we can extract an convergent subsequence from the $\theta_{j}^{(k)}$ yielding a curve $\tilde{\theta}$.

It remains to be shown that $\tilde{\theta}$ is an extremal of the Maupertuis variational principle. This is done as in ref. [20]. Assume it is not. Then in a sufficiently small neighbourhood of $\tilde{\theta}$, all curves are shortened by a finite positive amount. But $\tilde{\theta}$ is the limit of the $\theta^{(k)}$ and thus the amount by which the $\theta^{(k)}$ with large $k$ gets shortened tends to zero. This yields the desired contradiction. The smoothness of $\tilde{\theta}$ can for example be obtained from the fact that it is an extremal of the Maupertuis variational principle and thus the solution of a smooth system of ODE's. This concludes the proof.

\section{Proof of nonslinear stability}

\subsection{Proof of theorem 1.2}

We will prove the non-linear stability of the straight line configuration by showing that the energy

$E(\phi, \dot{\phi})=\frac{1}{2}(K(\phi) \dot{\phi}, \dot{\phi})+\mu^{2} W(\phi)$,

where $W(\phi)=(Q(\phi) I, I)^{-1}$, has a global nonde- generate minimum at $\phi=\dot{\phi}=\mathbf{0}$, corresponding to the straight chain. Theorem 1.2 is an immedjate consequence of the following two lemmas.

Lemma 4.1. At $\phi=\dot{\phi}=0, E(\phi, \dot{\phi})$ has a global minimum.

Lemma 4.2. The minimum $\phi=\dot{\phi}=0$ of the energy $E(\phi, \dot{\phi})$ is non-degenerate.

These lemmas will be proved at the end of this section. It is well known since Lagrange $[1,5]$ that a non-degenerate minimum of energy leads to stability. Nevertheless, we give full details in order to provide explicit bounds for a solution starting near the straight line configuration. We set $x=(\phi, \dot{\phi}) \in \mathbb{R}^{2 N}$ and subtract a constant from the energy $E$

$V(x)=E(x)-\mu^{2} W(0)$

in order to make $V(0)=0$. Letting $n=x /|x|$, $t=|x|$ for $x \neq 0$, we have by Taylor's formula

$$
V(x)=\left.\frac{1}{2} \frac{\mathrm{d}^{2}}{\mathrm{~d} \tau^{2}} V(\tau n)\right|_{\tau=0} t^{2}+\frac{1}{3 !} \frac{\mathrm{d}^{3}}{\mathrm{~d} \tau^{3}} V(\tau n) t^{3},
$$

for some $0 \leq \tau \leq t$. We can rewrite this as

$V(x)=(T x, x)+R(x)$,

with the matrix

$T=\left(\begin{array}{cc}\mu^{2} \mathrm{D}^{2} W(0) & 0 \\ 0 & K(0)\end{array}\right)$

and with the remainder

$$
\begin{aligned}
R(x) & =\frac{\mathrm{d}^{3}}{\mathrm{~d} \tau^{3}} V(\tau n) t^{3} \\
& =\sum_{i, j, k} V_{x_{i} x_{j} x_{k}}(\tau n) n_{i} n_{j} n_{k} t^{3},
\end{aligned}
$$

which satisfies the estimate

$$
|R(x)| \leq C(N, \mu)|x|^{2} \text { for }|x| \leq 1,
$$


where

$$
C(N)=N^{3} \max _{i, j, k} \sup _{|x| \leq 1}\left|V_{x_{i} x_{j} x_{k}}(x)\right|
$$

Denoting by $\lambda$ the minimal eigenvalue of $T$ and by $\Lambda$ its maximal eigenvalue, both positive, since $\mathrm{D}^{2} W$ and $K$ are both positive by lemma 4.2 , we obtain

$$
\begin{aligned}
& \frac{1}{2} \lambda|x|^{2} \leq V(x) \leq 2 \Lambda|x|^{2} \\
& \text { for }|x| \leq \min \left(1, \frac{\lambda}{2 C(N)}\right) .
\end{aligned}
$$

With this estimate it is now possible to prove stability of the straight configuration. Using the above estimate and the fact that $V(x(t))$ is constant along solutions, we see that

$$
|x(t)|^{2} \leq \frac{2}{\lambda} V(x)=\frac{2}{\lambda} V(x(0)) \leq 4 \frac{\Lambda}{\lambda}|x(0)|^{2}
$$

holds provided that $|x(t)| \leq \min (1, \lambda / 2 C(N))$ for all $t$. The latter is certainly achieved if

$|x(0)| \leq \frac{1}{2} \sqrt{\frac{\lambda}{\Lambda}} \min \left(1, \frac{\lambda}{2 C(N)}\right) \equiv \delta_{0}$.

This gives us a quantitative statement (which implies nonlinear stability). For any $|x(0)|<\delta_{0}$ given by (4.4) we have

$|x(t)|<2 \sqrt{\frac{\Lambda}{\lambda}}|x(0)| \quad$ for all $t \in \mathbb{R}$.

\subsection{Proof of lemma 4.1}

It suffices to prove that the moment of inertia $I(\phi)$ with respect to the center of mass has its unique global maximum at $\phi=0$ (fig. 2)

Let us mark the point $c_{0}$ on a link (or possibly a joint) where the center of mass lies when the chain is straightened out $(\theta=0)$. Having thus defined $c_{0}$ we look now at an arbitrary shape with $\phi \neq 0$ and (it suffices to) show that $I(\phi)<I(0)$. To that end we let $I_{0}(\phi)$ denote the moment of

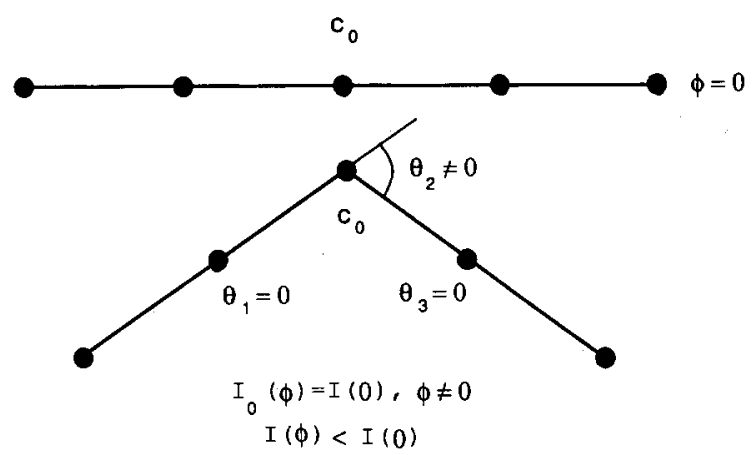

Fig. 2. Proving the straight chain $(\phi=0)$ maximizes the moment of inertia $I(\phi)$.

inertia relative to the point $c_{0}$ (thought of as a point marked on the chain), and show that

$I(\phi) \stackrel{(\mathrm{A})}{\leq} I_{0}(\phi) \stackrel{(\mathrm{B})}{\leq} I(0)$,

and moreover that at least one inequality (A) or (B) is strict.

The inequality (A) holds because the moment of inertia $I(\phi)$ relative to the center of mass is less than the moment of inertia $I_{0}(\phi)$ relative to any other point ( $c_{0}$ in this case), and strictly less if $c_{0}$ is not at the center of mass $c$. Indeed, denoting the positions of masses in some frame by $x_{i}$, we have

$c=\bar{x}=\frac{1}{N+2} \sum_{k=1}^{N+2} x_{i}$

and

$$
\begin{aligned}
I_{0}(\phi)= & m \sum\left(x_{i}-c_{0}\right)^{2} \\
= & m \sum\left[\left(x_{i}-\bar{x}\right)+\left(\bar{x}-c_{0}\right)\right]^{2} \\
= & m \sum\left(x_{i}-\bar{x}\right)^{2}+m \sum\left(\bar{x}-c_{0}\right)^{2} \\
& +2 m \sum\left(x_{i}-\bar{x}\right)\left(\bar{x}-c_{0}\right) \\
= & I(\phi)+\left(\bar{x}-c_{0}\right)^{2},
\end{aligned}
$$

thus proving (A).

Now (B) holds by the triangle inequality, according to which the distance of each mass from 
$c_{0}$ is maximized by $\phi=0$. If (B) is strict, we are done. We therefore assume that (B) is not strict. $I_{0}(\phi)=I(0), \phi \neq 0$, and prove then that $(\mathrm{A})$ is strict. We proceed as follows. Step 1: $I_{0}(\phi)=I(0)$ implies that the chain has not more than one bent link (and since $\phi \neq 0$, precisely one), i.e. that $\theta_{k} \equiv \phi_{k}-\phi_{k-1} \neq 0$ for one and only one $k$. Step 2: Precisely one bent link implies that (A) is strict. To carry out step 1 we note that $I(0)$ is the sum of squares of the distances form $c_{0}$ to each of the masses, while $I_{0}(\phi)$ is the sum of squares of distances from the same point $c_{0}$ to the masses when the chain is not straight. From the triangle inequality it is clear that the only way the two sums can be equal is when the distance from $c_{0}$ to each of the masses is maximized, i.e. each part of the chain lying between $c_{0}$ and any of the masses must be straight. Since $\phi \neq 0$, this shows that the only possibility left is that $c_{0}$ lies on a joint and that this and only this joint is not straight, as shown in fig. 2. This completes step 1. Step 2 is now very simple: we know now that the center of mass on the chain lies away from $c_{0}$, which by the above argument means that $I(\phi)<$ $I_{0}(\phi)$.

QED

\subsection{Proof of lemma 4.2-nondegeneracy of the minimum}

Since we already know that $(K(\phi) \dot{\phi}, \dot{\phi})$ is a positive definite quadratic form (see the end of section 2.4), we only need to show that at $\phi=0$, $\mathrm{D}^{2} W(0)$ is positive definite. To that end, using Taylor expansion of $W(\phi) \equiv 1 / I(\phi)$ around $\phi=$ 0 , it suffices to show that $\mathrm{D}^{2} I(0)$ is negative definite. We define $\hat{\phi}=\left(\phi_{0}, \phi\right) \in \mathbb{R}^{N+1}$ and

$\hat{I}(\hat{\phi})=(Q(\hat{\phi}) 1, I)=\sum_{k, l=0}^{N} c_{k l} \cos \left(\phi_{k}-\phi_{l}\right)$.

One has

$\hat{I}(0, \phi)=I(\phi)$
Computing the Hessian matrix $\left(\mathrm{D}^{2} \hat{I}(\hat{\phi})\right)_{n, m}=$ $\partial^{2} \hat{I} / \partial \phi_{n} \partial \phi_{m}, n, m=0, \ldots, N$, yields

$$
\begin{aligned}
& \left(\mathrm{D}^{2} \hat{I}(\hat{\phi}) \hat{\xi}, \hat{\xi}\right) \\
& \quad=2 \sum_{n, m=0}^{N} c_{n m} \cos \left(\phi_{n}-\phi_{m}\right)\left(\xi_{n} \xi_{m}-\xi_{n}^{2}\right),
\end{aligned}
$$

for any $\hat{\xi} \in \mathbb{R}^{N+1}$. Substituting $\hat{\phi}=0$ and using $c_{n m}>0$ and $\xi_{n} \xi_{m} \leq \frac{1}{2}\left(\xi_{n}^{2}+\xi_{m}^{2}\right)$ we obtain

$\left(\mathrm{D}^{2} \hat{I}(0) \hat{\xi}, \hat{\xi}\right) \leq 0$

Equality holds only when $\xi_{n}=\xi_{m}, n, m=$ $0, \ldots, N$. Variations of $\phi$ correspond to $\hat{\xi}=(0, \xi)$, $\xi \in \mathbb{R}^{N}$, resulting in the identity

$\left(\mathrm{D}^{2} \hat{I}(0) \hat{\xi}, \hat{\xi}\right)=\left(\mathrm{D}^{2} I(0) \xi, \xi\right)$

and hence $\left(D^{2} I(0) \xi, \xi\right) \leq 0$. As we have just observed, the equality holds only for $\xi_{n}=\xi_{m}$ which together with $\xi_{0}=0$ implies that $\xi_{n}=0$, for all $n$, so that $\phi=0$ is a nondegenerate minimum of $W(\phi)$.

\subsection{Proof of the instability of folded configurations}

We now prove that folded configurations (at least one $\theta_{j}=\pi$ ) are unstable. As before, it is sufficient to show that for such a configuration, the moment of inertia $I$ can be increased.

For this, we let $S_{1}$ be the part of the chain form one end till the first folded joint. Now either the center of mass of the rest of the chain lies to the right or to the left of the joint. (See fig. 3.) That is, either in part $\mathrm{A}$ or in part $\mathrm{B}$.

When the center of mass lies in part $A$, one is in a situation described by fig. 4 where $m_{1}$ represents the mass of $S_{1}$. Opening the first folded joint increases the distance between the center of masses and thus increases the moment of inertia.

When the center of mass lies in B, and possibly at the boundary between $\mathrm{A}$ and $\mathrm{B}$, let $S_{2}$ denote the part of the chain between the first folded joint and the next one. Now by assumption, the 


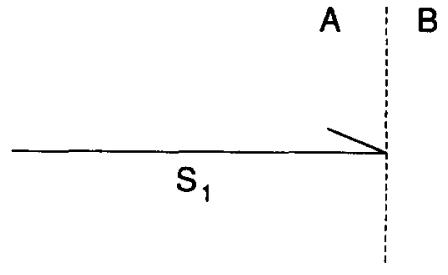

Fig. 3.

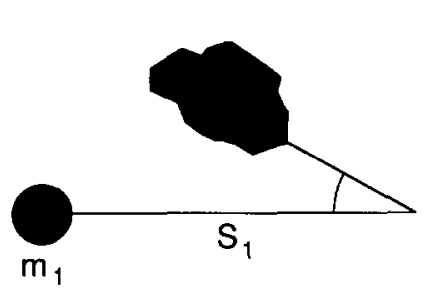

Fig. 4.

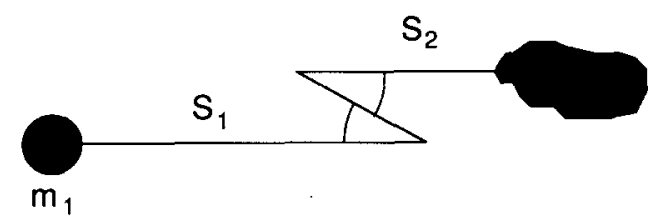

Fig. 5.

center of mass of the rest of the chain will lie in B so that one is in the situation described in fig. 5 . It is now easy to see that opening the first two folded joints in opposite directions will increase the moment of inertia. This shows that folded configurations are unstable.

\section{A remark on linearization and the Coriolis forces}

It is interesting to point out that the linearized equations for a straight line configuration contain no Coriolis forces. Indeed, linearizing the Euler-Lagrange equations around any solution $\phi(t)$, we obtain

$A(t) \ddot{\xi}+B(t) \dot{\xi}+C(t) \xi=0$,

where $A(t)=K(\phi(t)), \quad B(t)=\omega_{\phi}(\phi(t))-$ $\omega_{\phi}^{\mathrm{T}}(\phi(t)), C(t)=\frac{1}{2} \mu^{2} W^{\prime \prime}(\phi(t)) . A$ and $C$ are symmetric matrices, while $B(t)$ is skew-symmetric; it corresponds to the Coriolis term. Taking now a special solution $\phi=\left(\phi_{1}, \ldots, \phi_{N}\right)$ with $\phi_{j}=0$ or $\pi$, we obtain $B=0$, according to $(2.3 \mathrm{~b})$, while $A>0$. If $\phi=0$, then $C \equiv \frac{1}{2} \mu^{2} W^{\prime \prime}(\phi)>0$, as we have shown above, and the linearized system (5.1) is stable, as we know already from theorem 1.2. If, however, we consider a folded straight chain, i.e. if at least one of the components $\theta_{j}=\pi$, then $C$ is not positive definite, as is easy to show using the same method as proving lemma 4.1. It follows than that the linearization

$A \ddot{\xi}+C \xi=0$

around such a solution is unstable (and consequently so is the solution itself). To show that (5.2) is unstable, we let $\sqrt{A}>0$ be the positive definite square root of $A$, and let $\eta=\sqrt{A} \xi$; eq. (5.1) becomes

$\ddot{\eta}+D \eta=0$

where $D=(\sqrt{A})^{-1} C(\sqrt{A})^{-1}$. Since $(\sqrt{A})^{-1}$ is a symmetric matrix, $D$ is similar to $C$ and in particular $D$ has a negative eigenvalue, the same as $C$. This proves that (5.2) is unstable.

Conjecture. The number of negative eigenvalues of $C$, i.e. the number of positive eigenvalues of the Hessian $I^{\prime \prime}\left(\theta_{0}\right)$ of the moment of inertia equals the number of folds in the chain, i.e. the number of $j$ for which $\theta_{j}=\pi(\bmod 2 \pi)$.

\section{References}

[1] V.I. Arnol'd, Mathematical Methods of Classical Mechanics (Springer, Berlin, 1978).

[2] J. Baillieul, An enumerative theory of equilibrium rotations for planar kinematic chains, Contemp. Math., to appear.

[3] J. Baillieul and M. Levi, Rotational elastic dynamics, Physica D 27 (1987) 43-63.

[4] J. Baillieul and M. Levi, The nonlinear theory of rotational mechanics (1989), preprint.

[5] G. Birkhoff, Dynamical Systems, AMS Colloquium Publications, Vol. 9 (1927). 
[6] G. Birkhoff, Dynamical Systems with Two Degrees of Freedom, Trans. Am. Math. Soc. (1917) pp. 199-300.

[7] A.M. Bloch Stability and equilibria of deformable systems, in: Proc. 26th IEEE Conf. Dec. Contr. (Los Angeles, 1987) pp. 1443-1444.

[8] K.C. Chang, Y. Long and E. Zehnder, Forced Oscillations for the triple pendulum, in: Analysis etc. eds. P. Rabinowitz and E. Zehnder Academic Press, New York, (1990) pp. 177-209.

[9] P. Felmer, Multiple solutions for Lagrangian systems on $\mathrm{T}^{n}$, Madison preprint (1989).

[10] B. Horn, K. Hiokava and V. Vazirani, Dynamics of a three degree of freedom kinematic chain, MIT AI memo 478 (1977).

[11] F. Josellis, Forced oscillations on $\mathrm{T}^{n} \times \mathrm{R}^{n}$ having a prescribed rotation vector, preprint S.F.B. 237 (Bochum 1987).

[12] V.V. Koslov, Periodic Oscilations of a compound pendulum, Prikl. Math. Mekh. 44 (1980) 238-244.

[13] V.V. Kosiov, Calculus of variations in the large, Russian Math. Surv. 40 (1985) 37-71.

[14] P.S. Krishnaprasad and J.E. Marsden, Hamiltonian struc- ture and stability for rigid bodies with flexible attachments, Arch. Rat. Mech. Anal. 98 (1987) 71-93.

[15] M. Levi, Morse theory of an elastically perturbed rigid body, Contemp. Math. 97 (1989) 209-216.

[16] S. Laederich and M. Levi, Qualitative dynamics of planar chains, IMA preprint (1989).

[17] Y.G. Oh, N. Sreemath, P.S. Krishnaprasad and J.E. Marsden, The dynamics of coupled planar rigid bodies part II: Bifurcation, periodic orbits and chaos, J. Dynam. Diff. Eqs. (1989), to appear.

[18] T. Posbergh, J. Simo and J.E. Marsden, Stability analysis of a rigid body with attached geometrically nonlinear appendage by the energy-momentum method, Contemp. Math. (1989).

[19] E. Routh, The elementary part of a treatise on the dynamics of rigid bodies, Part 2, 4th Ed. (Macmillan, London) p. 788.

[20] H. Seifert and W. Threlfall, Variationsrechnung im Grossen (Chelsea, New York, 1948).

[21] E.A. Whittaker, Treatise on the Analytical Dynamics of Particles and Rigid Bodies, 4th Ed. (Dover, New York, 1944) par. 38. 